\title{
miR-150 promotes the proliferation and migration of non-small cell lung cancer cells by regulating the SIRT2/JMJD2A signaling pathway
}

\author{
KANQIU JIANG* , MINGJING SHEN*, YONGBING CHEN and WEIHUA XU \\ Department of Cardiothoracic Surgery, The Second Affiliated Hospital of Soochow University, \\ Suzhou, Jiangsu 215004, P.R. China
}

Received November 3, 2017; Accepted May 22, 2018

DOI: $10.3892 /$ or.2018.6487

\begin{abstract}
Lung cancer (LC), as the most common cause of cancer-related mortality worldwide, is characterized by difficulties in early detection, a high degree of malignancy, poor sensitivity to radiotherapy and chemotherapy, and a low 5-year survival rate. MicroRNA (miRNA) is a class of small, non-coding, endogenously expressed RNA that serves vital roles in RNA silencing and post-transcriptional regulation of gene expression. Previous studies have shown that abnormal expression of miRNA is relevant to various malignant tumors, including lung cancer. In the present study, miR-150 was found to be significantly upregulated in non-small cell lung cancer (NSCLC) cells, which also exhibited downregulation of SIRT2. Through downregulation of miR-150 and/or overexpression of SIRT2 in NSCLC cells (A549 and H1299), in vivo assays revealed that the suppression of miR-150 and re-expression of SIRT2 could inhibit NSCLC cell growth. Additionally, the present data demonstrated that miR-150 regulated NSCLC cell viability and mobility through SIRT2/JMJD2A. Finally, it was demonstrated that silencing of miR-150 led to inactivation of the AKT signaling pathway, which eventually inhibited the viability and mobility of NSCLC cells. This inhibitory effect of miR-150 could be exacerbated by upregulation of SIRT2. In conclusion, our results demonstrated that miR-150 plays an important role in the development of lung cancer by serving as an oncogene via the SIRT2/JMJD2A signaling pathway.
\end{abstract}

Correspondence to: Dr Weihua Xu, Department of Cardiothoracic Surgery, The Second Affiliated Hospital of Soochow University, 1055 Sanxiang Street, Gusu, Suzhou, Jiangsu 215004, P.R. China E-mail:wh_xu2016@aliyun.com

${ }^{*}$ Contributed equally

Key words: miR-150, SIRT2, JMJD2A, malignant phenotype, lung cancer

\section{Introduction}

Lung cancer (LC) is currently the second most frequent malignant tumor and the leading cause of mortality in both genders worldwide, with over 1.5 million cancer-related mortalities reported each year $(1,2)$. Based on histopathological differences, LC can be divided into two main types: Small cell lung cancer (SCLC; $15 \%$ of all lung cancers) and non-small cell lung cancer (NSCLC; 85\% of all lung cancers) (3). Despite the significant improvements that have been made in early diagnosis and therapeutic approaches, the survival rate of patients with LC is still far from satisfactory. LC is a highly heterogeneous malignant tumor, and thus clinical treatment strategy should be precise and personalized according to each patient's clinical features. In recent years, a number of systematic studies on novel molecular and gene markers involved in the pathogenesis of LC have been conducted (4-6). However, evidence for the underlying mechanisms involved in the carcinogenesis and development of LC remains limited.

MicroRNAs (miRNA/miRs) are small, non-coding RNA molecules of approximately 22 nucleotides in length, which bind to specific complimentary recognition sequences in the 3'-untranslated regions (UTRs) of target mRNAs, to thereby function in RNA silencing and post-transcriptional regulation of gene expression $(7,8)$. While the majority of miRNAs are located within the cell, certain miRNAs, commonly known as circulating miRNAs or extracellular miRNAs, have also been found in extracellular environments, including in various biological fluids and cell culture media. miRNAs have been proven to play important roles in many biological behaviors of tumor cells, including cell survival, proliferation, mobility, apoptosis, metabolism and other pathological features of various tumors (9). Abnormal expression of miRNAs is relevant to almost all types of tumor, and miRNAs may function as both oncogenes and tumor suppressors during tumor progression $(10,11)$. For instance, miR-224 may promote the malignant progression of NSCLC by partially antagonizing the functions of SMAD4 and TNFAIP1 (12). miR-31 is reported to be relevant to prognosis, survival time and distant metastasis in patients with lung adenocarcinoma (13). miR-150 has been proven to locate on chromosome 19q13 and participate in hematopoiesis (14). Further evidence has demonstrated that 
miR-150 is a crucial regulatory factor in tumor progression. In a previous study, miR-150 promoted gastric cancer cell proliferation by negatively regulating the proapoptotic gene EGR2 (15). miR-150 increased the viability and mobility of LC cells by targeting SRC kinase signal inhibitor 1 or p53 $(16,17)$. These studies revealed that miRNAs have the potential to be used as targets in the treatment of different cancers. In particular, miR-150 may be crucial in the progression of various cancers, including LC. However, to date, the exact molecular mechanism of miR-150 in regulating the tumorigenesis of LC has remained poorly understood.

In the present study, our aim was to investigate the function of miR-150 in NSCLC cells, and to elucidate the possible underlying mechanisms. The present data identified that the expression of miR-150 was dramatically upregulated in NSCLC cells. Furthermore, miR-150 was found to regulate the expression levels of SIRT2 and JMJD2A. Moreover, miR-150 was also identified to function in the viability and mobility of NSCLC cells via regulating SIRT2/JMJD2A expression. Notably, in vivo assays revealed that reduced miR-150 expression and/or re-expression of SIRT2 inhibited NSCLC cell growth. Finally, results revealed that miR-150 regulated SIRT2/JMJD2A expression by activating the AKT signaling pathway. Therefore, the miR-150-SIRT2/JMJD2A axis could be a promising molecular target in therapeutic strategies aimed at preventing the malignant progression of NSCLC.

\section{Materials and methods}

Cell lines and cell culture. A human normal lung cell line (MRC-5) and four human NSCLC cell lines (A549, H460, H1299 and H520) were purchased from the Cell Bank of the Chinese Academy of Sciences (Shanghai, China). These cells were cultured in Dulbecco's modified Eagle's medium (DMEM; Gibco; Thermo Fisher Scientific, Inc., Waltham, MA, USA) supplemented with $10 \%$ fetal bovine serum (FBS; HyClone Laboratories; GE Healthcare Life Sciences, Logan, UT, USA), $100 \mathrm{U} / \mathrm{ml}$ penicillin, $100 \mu \mathrm{g} / \mathrm{ml}$ streptomycin and $1 \%$ glutamine (Invitrogen; Thermo Fisher Scientific, Inc.). All cells were maintained at $37^{\circ} \mathrm{C}$ with $5 \% \mathrm{CO}_{2}$.

Cell infection and transfection. For miR-150 downregulation or overexpression, miR-150 inhibitor (LNA-anti-miR-150) (Exiqon, Vedbæk, Denmark) or miR-150 mimics (Invitrogen; Thermo Fisher Scientific, Inc.) were respectively added to the culture medium as in a previous study (18). The transfection medium was replaced at $4 \mathrm{~h}$ post-transfection with regular culture medium.

For SIRT2 overexpression or knockdown, A549 and H1299 cell lines were infected with adenovirus expressing SIRT2 (Ad-SIRT2) or retrovirus expressing sh-STRT2, respectively, as in our previous study (19).

Reverse transcription-quantitative PCR (RT-qPCR). Real-time RT-qPCR was used to detect the expression level of miR-150 by a MiniOpticon $^{\text {TM }}$ Two-Color Real-Time PCR Detection system (Bio-Rad Laboratories, Hercules, CA, USA). miRNA was extracted from cultured cells by using an Applied Biosystems mirVana miRNA Isolation kit (Thermo Fisher Scientific, Inc.). U6 was used as the internal control.
Western blotting. Total protein isolated from $10^{5}$ A549 and H1299 cells was lysed with RIPA lysis buffer (Beyotime Institute of Biotechnology, Haimen, China) and detected by a western blot assay as previously described (18). Antibodies against the following target proteins were used: Phosphorylated-AKT ${ }^{\text {Ser473 }}$ (dilution 1:1,000; cat. no. sc-7985-R), AKT (dilution 1:1,000; cat. no. sc-8312), JMJD2A (dilution 1:500; cat. no. sc-81302), SIRT2 (dilution 1:1,000; cat. no. sc-135793; Santa Cruz Biotechnology, Santa Cruz, CA, USA), mouse PCNA (dilution 1:1,000; cat. no. ab-18197; Abcam, Cambridge, MA, USA) and GAPDH (dilution 1:5,000; Kangchen Bio-tech, Shanghai, China); the secondary antibodies were sheep anti-mouse IgG and anti-rabbit IgG (dilution 1:6,000; R\&D Systems China, Shanghai, China). GAPDH was used as the internal control. All protein bands were semi-quantitatively detected with ImageJ software (National Institutes of Health, Bethesda, MD, USA).

MTT assay. A549 and H1299 cell viability was monitored using a Cell Proliferation kit I (MTT) (cat. no. 11465007001; Sigma-Aldrich; Merck KGaA, Darmstadt, Germany) according to the manufacturer's instructions.

Plate colony formation assay. NSCLC cell lines A549 and H1299 were collected following transfection, and $1 \times 10^{3}$ cells were mixed with high-glucose DMEM supplemented with $10 \%$ fetal bovine serum (FBS) and cultured in 6-well tissue culture plates. The cells were continuously cultured for 2 weeks. Following culture, all cell colonies were stained with $0.005 \%$ crystal violet dye liquor. The stained cells were observed by microscopy (Leica DM2500; Leica Microsystems, Wetzlar, Germany) and 10 random fields were selected for cell colony counting. The experiment was conducted three times.

Cell mobility assays. Cell mobility assays were performed with a Transwell chamber (EMD Millipore, Billerica, MA, USA) according to the manufacturer's guidelines. For an invasion assay, a total of $5 \times 10^{4}$ A549 and H1299 cells at 24 h post-transfection were plated onto an $8-\mu \mathrm{m}$ pore size Transwell insert pre-coated with extracellular matrix (ECM) (1:6 mix with DMEM) (BD Biosciences, San Jose, CA, USA). A cell migration assay was performed using the Transwell insert without the pre-coating with ECM. After conventional cell culture for $48 \mathrm{~h}$, all cells which had adhered to the upper surface of the Transwell insert were wiped away gently with a cotton swab. The remaining cells which had passed through the Transwell insert were stained with $0.005 \%$ crystal violet dye liquor for $15 \mathrm{~min}$. The stained cells were observed by microscopy (Leica DM2500; Leica Microsystems) and 10 random fields were selected for cell counting. The experiment was conducted three times.

Tumor xenograft experiments. Xenograft mouse experiments were performed as previously described (18). Twenty-four male $\mathrm{BALB} / \mathrm{c}$ nude mice ( $20 \mathrm{~g}$ weight) at 8 weeks of age (Shanghai SLAC Laboratory Animal Center of Chinese Academy of Sciences, Shanghai, China) were randomly divided into four groups ( $\mathrm{n}=6 /$ group), subcutaneously injected the parental, LNA-anti-miR150-transfected, Ad-SIRT2-transfected and both transfected A549 cells, respectively, into the right 

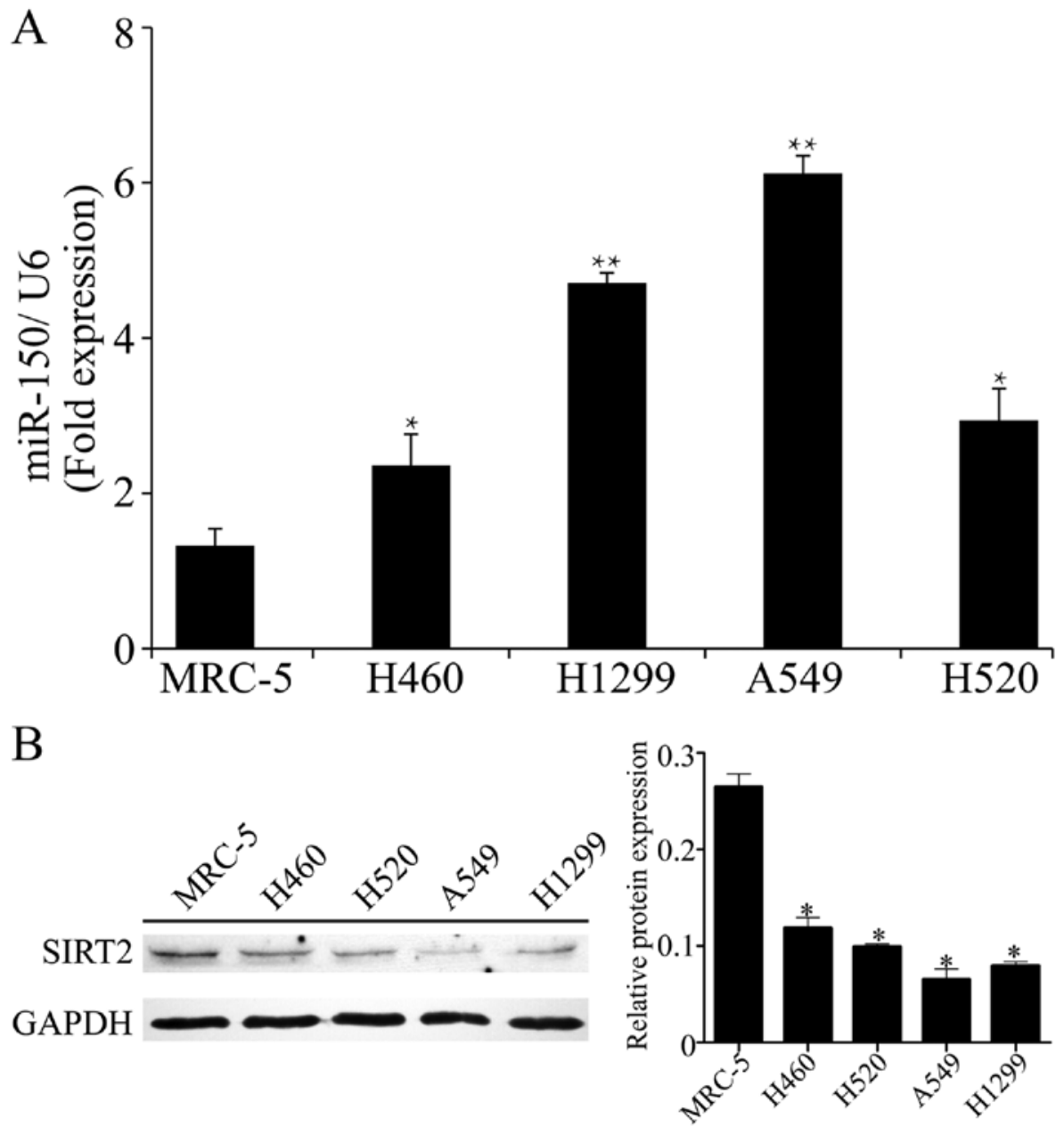

Figure 1. miR-150 overexpression in NSCLC cell lines is accompanied by suppression of SIRT2. (A) The expression of miR-150 was examined by real-time RT-qPCR, with the results normalized to U6 expression. ${ }^{*} \mathrm{P}<0.05,{ }^{* *} \mathrm{P}<0.001$ vs. normal control cell line MRC-5; (B) Detection of SIRT2 protein expression by western blotting. Expression levels of SIRT2 were semi-quantitatively analyzed with ImageJ software, with normalization to the corresponding GAPDH density. ${ }^{*} \mathrm{P}<0.01$ vs. normal control cell line MRC-5.

and left flanks of male nu/nu mice. Mice were maintained in pathogen-free conditions and cared for according to the Laboratory Animal Care guidelines. They were given radiation-sterilized food pellets and distilled water. Tumor size was measured regularly, and tumor volume was estimated with the formula: $a \times b^{2} \times 0.5$, in which a and $b$ represent the maximal and minimal diameters. Mice were sacrificed by anesthetization at the end of the observation period and cancer tissues were harvested and the tumor weights were measured. Then, the tumors were fixed in $10 \%$ neutral formaldehyde for $6 \mathrm{~h}$, and consecutive paraffin-embedded sections were cut to examine the expression of PCNA, JMJD2A and p-AKT ${ }^{\text {Ser473 }}$ by immunohistochemistry. The animal welfare guidelines for the care and use of laboratory animals were followed and the experimental protocol was approved by the Animal Care Committee of the Second Affiliated Hospital of Soochow University.

Statistical analysis. The experimental data from three independent experiments were presented as the mean \pm standard deviation (SD) and analyzed using one-way analysis of variance (ANOVA) test with post hoc contrasts by the Student-Newman-Keuls test. The software package PASW
Statistics 18.0 (SPSS, Inc., Chicago, IL, USA) was used for statistical analysis. A $\mathrm{P}<0.05$ was considered to indicate statistical significance.

\section{Results}

miR-150 is overexpressed in NSCLC cell lines and is accompanied by suppression of SIRT2. Our previous studies revealed that miR-150 was obviously upregulated in NSCLC tissues compared with that noted in normal tissues. To confirm the expression of miR-150 in NSCLC cells, we performed RT-qPCR to detect the expression of miR-150 in four NSCLC cell lines. As shown in Fig. 1A, compared with the normal cell line MRC-5, miR-150 was significantly upregulated in the four NSCLC cells lines. In particular, the relative expression of miR-150 was highly increased in the A549 and H1299 cells (6.12 \pm 0.23 in $\mathrm{A} 549,4.71 \pm 0.13$ in $\mathrm{H} 1299 ; \mathrm{P}<0.01$ vs. normal cell line). Meanwhile, significant suppression of SIRT2 was observed in the NSCLC cell lines, particularly in A549 and H1299 cells ( $\mathrm{P}<0.01$ vs. normal cell line; Fig. 1B). Accordingly, the A549 and H1299 cell lines, with obvious upregulation of miR-150 and downregulation of SIRT2, were selected for the following assays. 

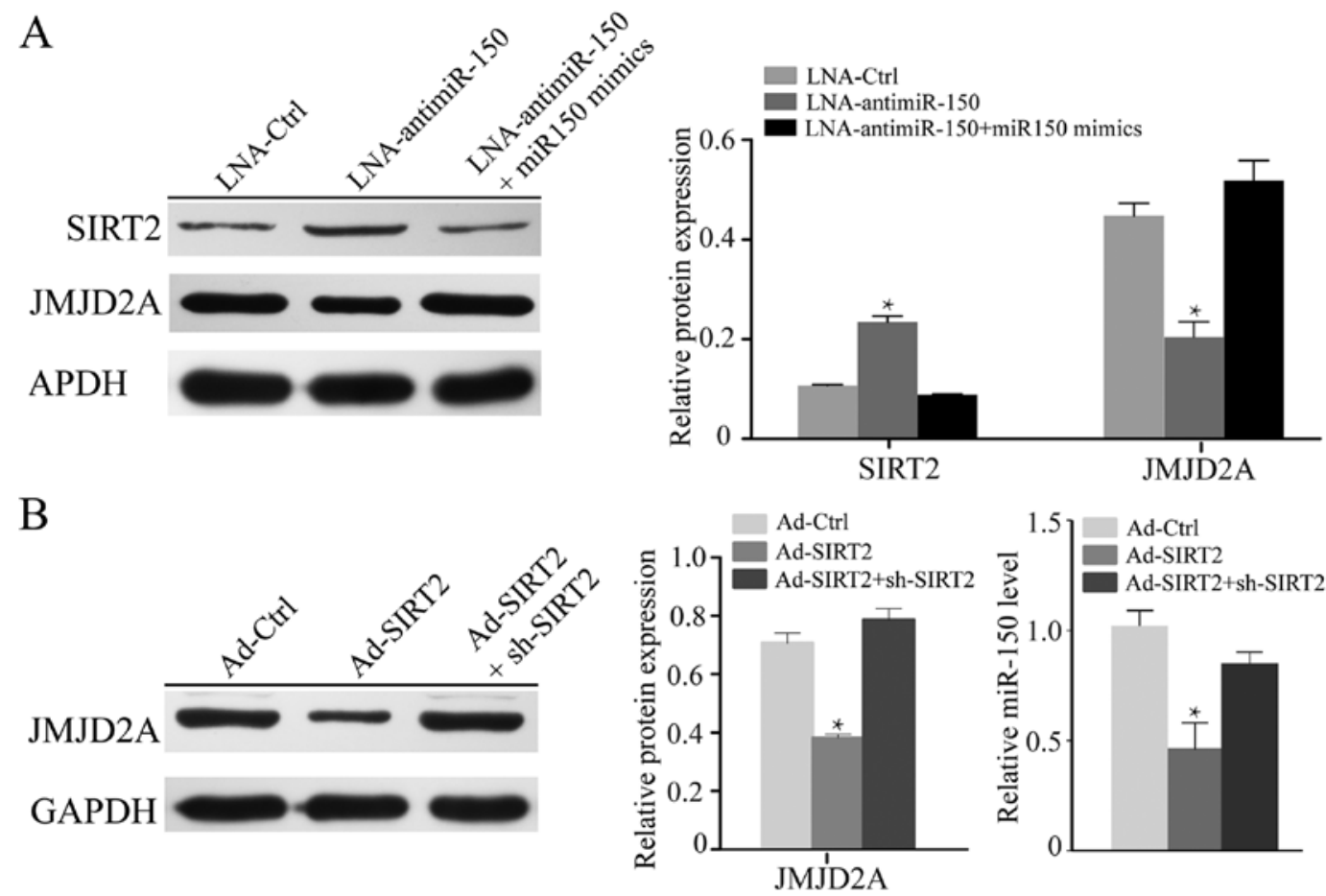

Figure 2. miR-150 affects the expression of SIRT2 and JMJD2A in NSCLC cells. (A) H1299 cells were cultured in 6-well plates transfected with LNA-antimiR-150, and then transfected with miR-150 mimics to rescue the level of miR-150, and $48 \mathrm{~h}$ later, the protein expression of SIRT2 and JMJD2A was examined by western blot analysis. Semi-quantitative analysis of SIRT2 and JMJD2A was then performed, with normalization to corresponding GAPDH density. " $\mathrm{P}<0.05$ vs. negative vector control group. (B) Detection of JMJD2A protein expression by western blotting in H1299 cells infected with adenovirus Ad-SIRT2. Expression levels of JMJD2A were semi-quantitatively analyzed with ImageJ software, with normalization with the corresponding GAPDH density. " $\mathrm{P}<0.05$ vs. normal control cell line MRC-5. miR-150 expression was detected by real-time RT-qPCR, with the results normalized to miR-150 expression in the negative vector control group. "P<0.05 vs. negative vector control group.

miR-150 effects the expression of SIRT2 and JMJD2A in NSCLC cell lines. Previous studies have demonstrated that miR-150 expression is markedly positively related to the JMJD2A level, while being significantly negatively correlated with SIRT2 and JMJD2A expression in A549 cells $(18,19)$. To further investigate the effect of miR-150 on H1299 cells, LNA-anti-miR-150 was used to silence miR-150, and miR-150 mimics were used to recover the level of miR-150. The knockdown of miR-150 in H1299 cells notably increased the expression of SIRT2 and reduced the level of JMJD2A $(\mathrm{P}<0.05$; Fig. 2A). In turn, upon transfection with miR-150 mimics, the normal expression levels of SIRT2 and JMJD2A were recovered. To further explore the relationship between miR-150 and SIRT2, we knocked down or overexpressed SIRT2 in H1299 cells. As shown in Fig. 2B, re-expression of SIRT2 suppressed the expression of JMJD2A and miR-150 $(\mathrm{P}<0.05)$.

Downregulation of miR-150 combined with upregulation of SIRT2 significantly inhibits the proliferation and neoplastic capacity of NSCLC cell lines. To explore the effect of miR-150 downregulation and SIRT2 re-expression on the proliferation of NSCLC cells, A549 and H1299 cell proliferation were measured after transfection with LNA-anti-miR-150 or Ad-SIRT2. At $48 \mathrm{~h}$ after transfection, the proliferation rate of cells transfected with LNA-anti-miR-150 or Ad-SIRT2 was decreased, relative to that of the negative controls. Moreover, combined treatment with LNA-anti-miR-150 and Ad-SIRT2 showed the most significant inhibitory effect on cell proliferation in A549 and H1299 cells (P<0.05; Fig. 3A). Next, the neoplastic capacity of NSCLC cells was examined by plate colony formation assay. The results demonstrated that the cells transfected with LNA-anti-miR-150 or Ad-SIRT2 formed less colonies than the corresponding control groups $(\mathrm{P}<0.05$; Fig. 3B). Similarly, the A549 and H1299 groups co-transfected with LNA-anti-miR-150 and Ad-SIRT2 exhibited the most obvious suppression of neoplastic capacity ( $\mathrm{P}<0.001$; Fig. 3B). In addition, western blot analysis was used to test the ability of miR-150 downregulation and SIRT2 re-expression to regulate the expression of PCNA (proliferating cell nuclear antigen), which serves an important role in the proliferation of cells. The data indicated that PCNA protein expression was reduced in the LNA-anti-miR-150 or Ad-SIRT2-transfected cells, but remained to be expressed to a high level in the respective negative control groups $(\mathrm{P}<0.05$; Fig. 5). Furthermore, significant reduction of PCNA expression was observed in the NSCLC cells co-transfected with LNA-anti-miR-150 and Ad-SIRT2 ( $\mathrm{P}<0.001$; Fig. 5). These results demonstrated that the combination of downregulated miR-150 and upregulated SIRT2 could strongly inhibit the proliferation and neoplastic capacity of NSCLC cells.

Downregulation of miR-150 combined with upregulation of SIRT2 obviously reduces cellular motility in NSCLC cell lines. To investigate the effect of miR-150 downregulation and SIRT2 re-expression on cellular motility, the NSCLC cell lines A549 and H1299 were subjected to Transwell cellular mobility assays after transfection with LNA-anti-miR-150 and/or Ad-SIRT2. The results indicated that the relative migrated 
A

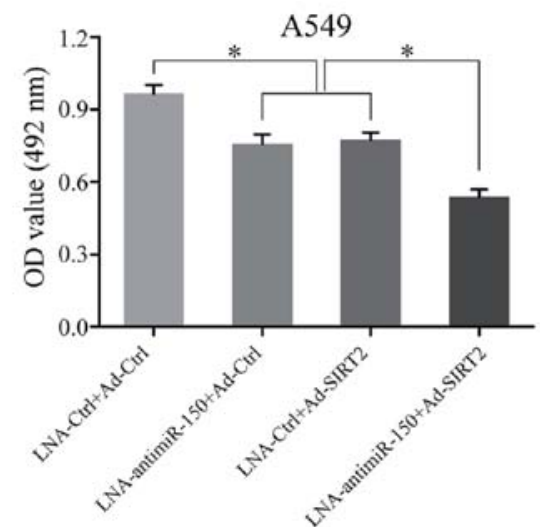

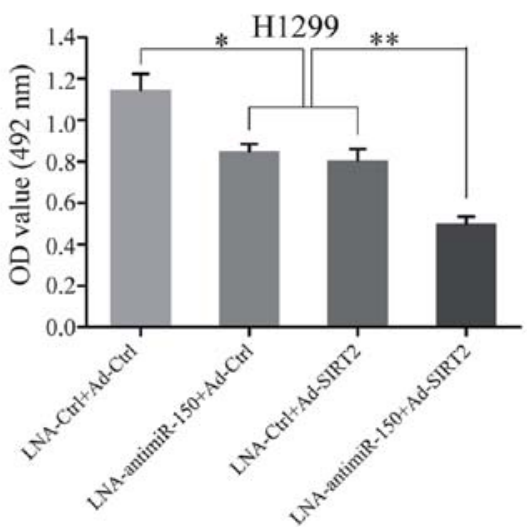
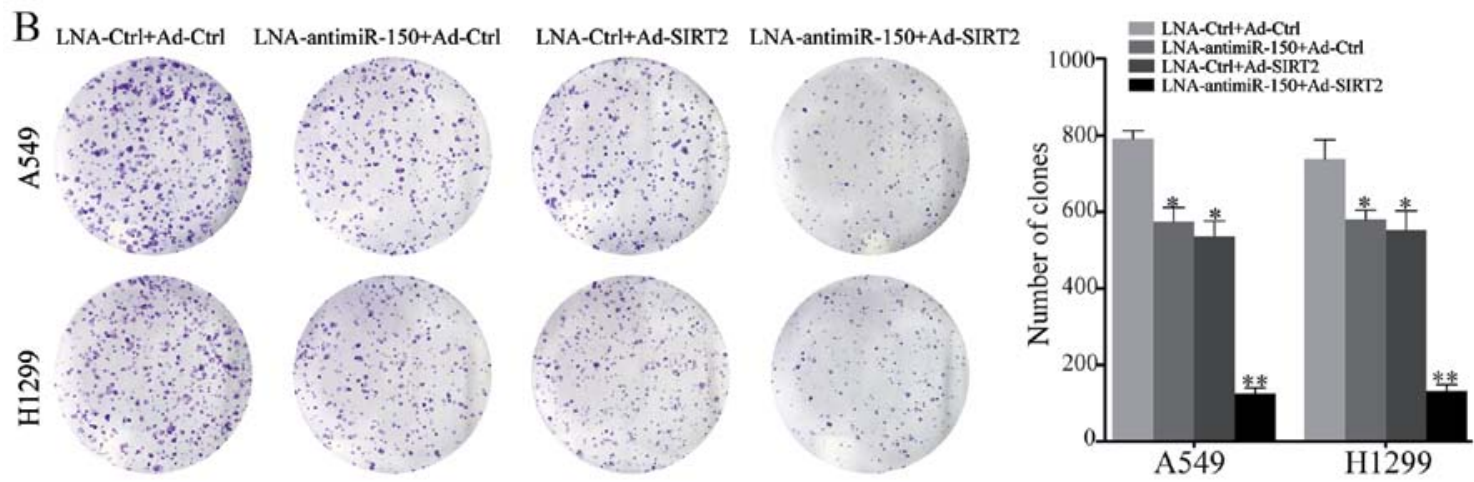

Figure 3. Downregulation of miR-150 combined with upregulation of SIRT2 significantly inhibits the proliferation and neoplastic capacity of NSCLC cell lines. (A) The cell viability of A549 and H1299 cells transfected with LNA-anti-miR-150 or Ad-SIRT2 or both vectors was measured with a cell proliferation kit. "P $<0.05,{ }^{* *} \mathrm{P}<0.01$; (B) The neoplastic capacity of A549 and H1299 cells transfected with LNA-anti-miR-150 or Ad-SIRT2 or both vectors was assessed by a plate colony formation assay. Cell colonies were counted and analyzed in a histogram. ${ }^{*} \mathrm{P}<0.05,{ }^{* *} \mathrm{P}<0.01$ vs. negative vector control group.
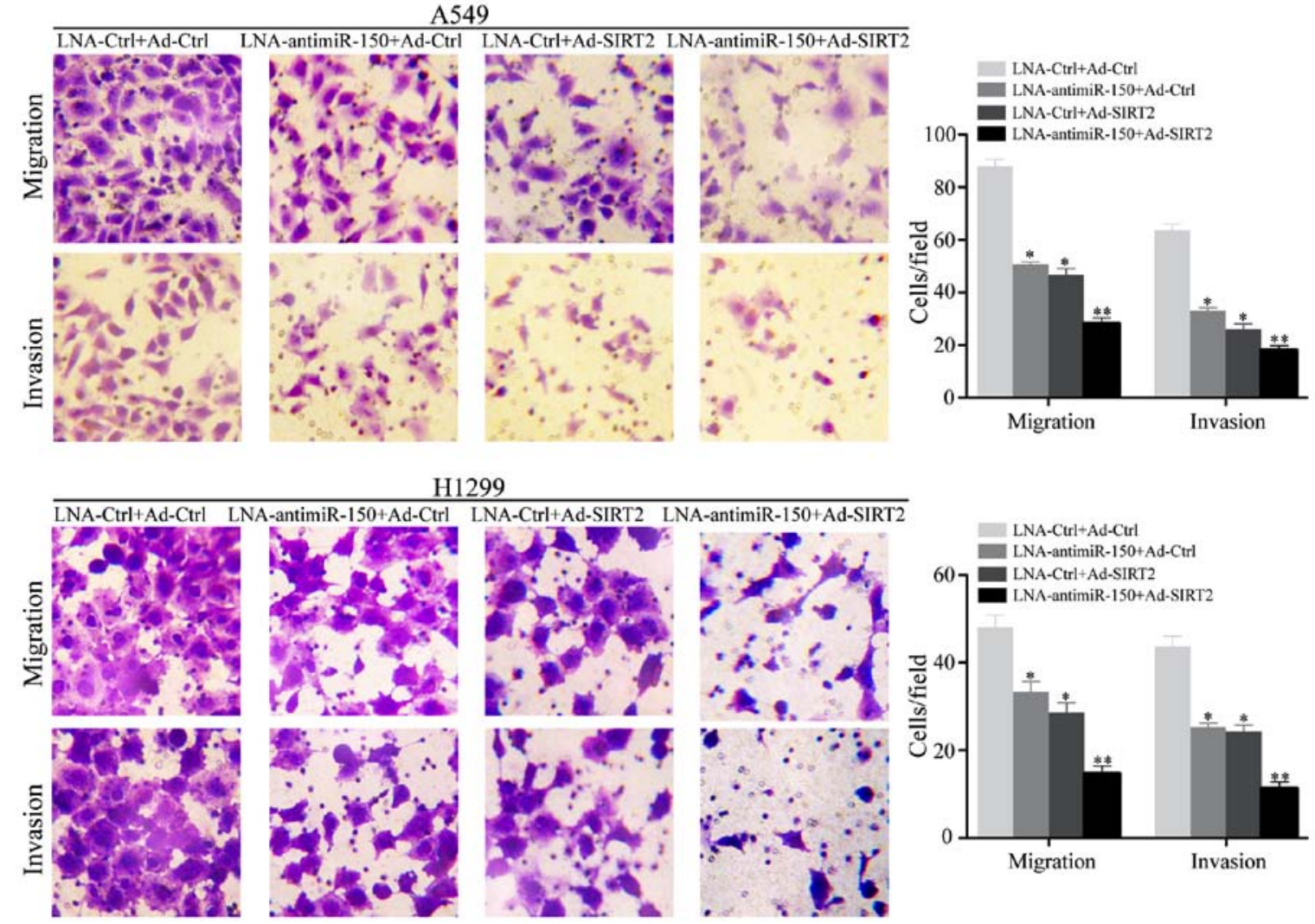

Figure 4. Downregulation of miR-150 combined with upregulation of SIRT2 obviously reduces cellular motility in NSCLC cell lines. The migratory and invasive properties of A549 and H1299 cells transfected with LNA-anti-miR-150 or Ad-SIRT2 or both vectors were assessed by cell mobility assays in Transwell plates (original magnification, x100). The migrated cells were counted and analyzed in a histogram; Error bars correspond to the standard deviation of the mean, $\mathrm{n}=3 .{ }^{*} \mathrm{P}<0.05,{ }^{* *} \mathrm{P}<0.01$ vs. negative vector control group. 

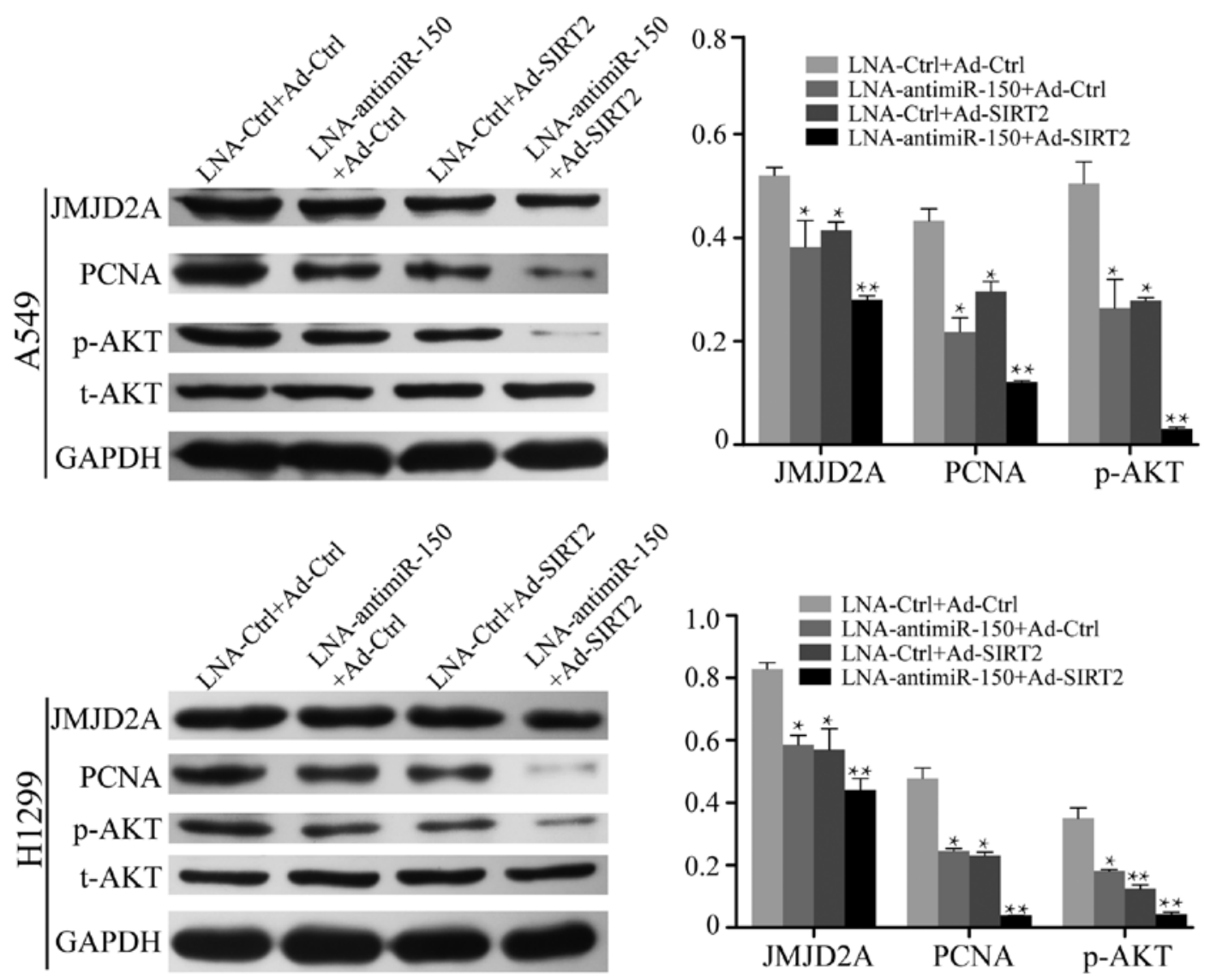

Figure 5. Reduction of miR-150 and re-expression of SIRT2 leads to suppression of JMJD2A and inactivation of the AKT signaling pathway in NSCLC cell lines. Detection of JMJD2A, PCNA and AKT pathway-related protein expression by western blotting. Expression levels of JMJD2A, PCNA, p-AKT ${ }^{\text {err473 }}$ and AKT were semi-quantitatively analyzed with ImageJ software, with normalization to the corresponding GAPDH density. ${ }^{*} \mathrm{P}<0.05,{ }^{* *} \mathrm{P}<0.01$ vs. negative vector control groups.

and invaded cell numbers were obviously decreased in the cell groups transfected with LNA-anti-miR-150 or Ad-SIRT2, compared with the negative controls $(\mathrm{P}<0.05$; Fig. 4). In particular, the group transfected with both LNA-anti-miR-150 and Ad-SIRT2 showed the lowest numbers of migrated and invaded cells $(\mathrm{P}<0.001 ;$ Fig. 4).

Reduction of miR-150 and re-expression of SIRT2 leads to suppression of JMJD2A and inactivation of the AKT signaling pathway in NSCLC cell lines. It has been well demonstrated that NSCLC cells exhibit high expression of miR-150 and low expression of SIRT2. To further investigate the effect of miR-150 suppression and SIRT2 re-expression on NSCLC cells, LNA-anti-miR-150 and Ad-SIRT2 were transfected into A549 and H1299 cells, and the relative expression levels of AKT and JMJD2A were measured by western blot analysis. The results demonstrated that the expression of JMJD2A was decreased in the cell groups transfected with LNA-anti-miR-150 or Ad-SIRT2 $(\mathrm{P}<0.05$; Fig. 5). The inhibition of JMJD2A expression was more marked in cells co-transfected with LNA-anti-miR-150 and Ad-SIRT2 ( $\mathrm{P}<0.001$; Fig. 5). In addition, reduction of miR-150 or re-expression of SIRT2 decreased the expression of p-AKT ${ }^{\text {Ser473 }}$ in A549 and H1299 cells ( $<<0.05$; Fig. 5). Co-treatment to reduce miR-150 expression and re-express SIRT2 further inhibited p-AKT ${ }^{\text {Ser473 }}$ expression in the NSCLC cells $(\mathrm{P}<0.001 ;$ Fig. 5). These results suggested that reduction of miR-150 and re-expression of SIRT2 leads to suppression of JMJD2A and effectively inactivates the AKT signaling pathway.

Reduction of miR-150 and re-expression of SIRT2 leads to suppression of NSCLC tumor growth in vivo. To further explore the effect of miR-150 on the NSCLC cells, a xenograft tumor growth assay was performed. The results showed that miR-150 knockdown or SIRT2 overexpression significantly suppressed NSCLC tumor growth in vivo (Fig. 6A and B; $\mathrm{P}<0.05)$. Moreover, co-transfection of A549 cells with miR-150 knockdown and SIRT2 re-expression vectors could further inhibit NSCLC tumor growth in vivo (Fig. 6A and B; $\mathrm{P}<0.001)$. Next, the paraffin-embedded tumor sections were examined immunohistochemically. The results showed that the expression of JMJD2A, PCNA and p-AKT ${ }^{\mathrm{Ser} 473}$ were decreased in the groups injected with cells transfected with LNA-anti-miR-150 or Ad-SIRT2. Moreover, the expression of JMJD2A, PCNA and p-AKT ${ }^{\text {Ser473 }}$ were more significantly inhibited in the group injected with cells co-transfected with LNA-anti-miR-150 and Ad-SIRT2 (Fig. 6C). These results suggested that reduction of miR-150 and re-expression of SIRT2 could inhibit NSCLC growth in vivo. It also provided evidence that miR-150 may play a tumor-promoting role in lung cancer. 

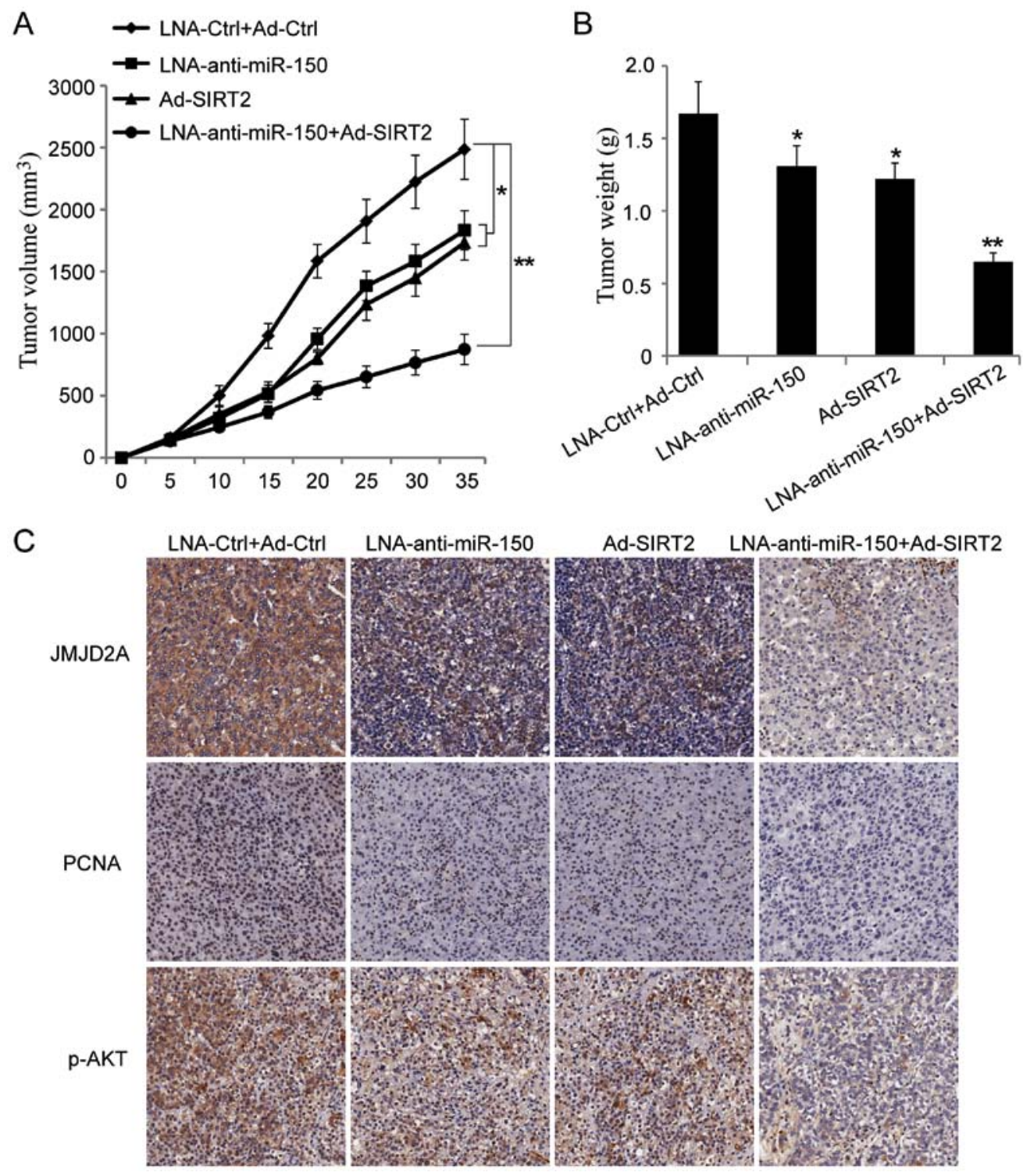

Figure 6. Reduction of miR-150 and re-expression of SIRT2 leads to suppression of NSCLC tumor growth in vivo. (A) The suppressive effect of miR-150 knockdown and SIRT2 re-expression on NSCLC tumor growth was analyzed, relative to the negative group, ${ }^{*} \mathrm{P}<0.05,{ }^{* * *} \mathrm{P}<0.01$. (B) Comparison of tumor weight ( $\mathrm{n}=6$ in each group). ${ }^{*} \mathrm{P}<0.05,{ }^{* *} \mathrm{P}<0.01$. (C) The expression of PCNA, JMJD2A and $\mathrm{p}-\mathrm{AKT}{ }^{\mathrm{Ser} 473}$ were examined by immunohistochemistry.

\section{Discussion}

Abnormal expression of miRNAs is relevant to almost all types of malignant tumor, and miRNAs can function both as oncogenes and tumor suppressors during tumor progression. To date, several miRNAs have been identified to play vital roles in LC pathogenesis, and could be novel target molecules for early diagnosis and clinical treatment (20-23).

miR-150 was first identified as a hematopoietic cell-specific miRNA that participated in hematopoiesis (24). Increasing evidence has demonstrated that miR-150 may also be involved in the malignant progression of various human tumors. More notably, miR-150 may function as an oncogene or a tumor suppressor in different tumors, dependent on its expression level and target genes in specific tumors. Recently, the biological functions of miR-150 in relation to the malignant phenotypes of cancer cells have been reported, and a series of target genes have been identified. In breast cancer,
miR-150 promotes the proliferation and malignant behavior (including migration, invasion and apoptosis resistance) of tumor cells by targeting the pro-apoptotic purinergic $\mathrm{P} 2 \mathrm{X} 7$ receptor (25). In gastric cancer cells, miR-150 could promote cell growth by negatively regulating the pro-apoptotic gene EGR2 in vitro (15). Moreover, miR-150 inhibited tumor cell metastasis in esophageal squamous cell carcinoma and hepatocellular carcinoma by targeting ZEB1 and GAB1, respectively $(26,27)$. However, to date, the exact molecular mechanism of miR-150 in the malignant progression of NSCLC remains poorly understood.

Our previous studies have revealed that miR-150 was upregulated in NSCLC tissues and positively related to the expression of JMJD2A. In turn, JMJD2A was able to regulate cell growth and apoptosis in an miR-150-dependent manner in NSCLC. Meanwhile, SIRT2, as an anti-oncogenic protein, exhibited downregulated expression in NSCLC. Additionally, SIRT2 could combine to the promoter region of JMJD2A 
and negatively regulated the expression of JMJD2A $(18,19)$. Based on this previous evidence, we speculated that miR-150 may affect the malignant progression of NSCLC cells by regulating SIRT2/JMJD2A. Our present data revealed that miR-150 was overexpressed in NSCLC cells compared with that noted in normal lung cells. Notably, NSCLC cell lines that highly expressed miR-150 exhibited downregulation of SIRT2. Moreover, silencing of miR-150 in NSCLC cells obviously promoted the expression of SIRT2 and reduced the level of JMJD2A, which could be recovered by transfection with miR-150 mimics. To further explore the relationship between miR-150 and SIRT2, we knocked down or overexpressed SIRT2 in NSCLC cells. Our results revealed that the re-expression of SIRT2 could suppress the expression of JMJD2A and miR-150. To further investigate whether miR-150 regulated NSCLC viability and mobility via SIRT2/JMJD2A, we knocked down miR-150 and overexpressed SIRT2 respectively or simultaneously in NSCLC cells. The silencing of miR-150 inhibited cell viability, while overexpressing-SIRT2 aggravated the inhibitory effect on cell proliferation, migration and invasion, suggesting that miR-150 was essential for the function of SIRT2/JMJD2A in NSCLC. These results suggested that miR-150 regulated NSCLC viability and mobility by regulating SIRT2/JMJD2A. Additionally, the silencing of miR-150 or re-expression of SIRT2 decreased the expression of PCNA and p-AKT Ser473 in NSCLC cells; furthermore, co-treatment to knockdown miR-150 and re-express SIRT2 could further inhibit PCNA and p-AKT ${ }^{\text {Ser473 }}$ expression in the NSCLC cells. Meanwhile, in vivo results of a tumor xenograft model demonstrated that miR-150 suppression and SIRT2 re-expression could inhibit NSCLC growth, and provided evidence that miR-150 may play a tumor-supporting role in lung cancer. Finally, our results suggested that reduction of miR-150 and re-expression of SIRT2 may lead to suppression of JMJD2A and effectively inactivate the AKT signaling pathway. Further studies are now needed to elucidate the signaling pathways involved in more detail.

In conclusion, the present study demonstrates that miR-150 may contribute to the malignant phenotype in NSCLC by regulating SIRT2/JMJD2A. miR-150 alone or in combination with SIRT2 may serve as a potential therapeutic target in NSCLC.

\section{Acknowledgements}

Not applicable.

\section{Funding}

The present study was supported by grants from the Science and Technology Development Project of Suzhou Municipality, Jiangsu Province (grant no. SYSD2016090) and the Science and Technology Bureau of Suzhou Municipality, Jiangsu Province (grant no. SYS201719).

\section{Availability of data and materials}

The datasets used during the present study are available from the corresponding author upon reasonable request.

\section{Authors' contributions}

YC and WX conceived and designed the experiments; $\mathrm{KJ}$ and MS performed the experiments; KJ analyzed the data; KJ and WX wrote the study. All authors read and approved the manuscript and agree to be accountable for all aspects of the research in ensuring that the accuracy or integrity of any part of the work are appropriately investigated and resolved.

\section{Ethics approval and consent to participate}

The animal welfare guidelines for the care and use of laboratory animals were followed and the experimental protocol was approved by the Animal Care Committee of the Second Affiliated Hospital of Soochow University.

\section{Patient consent for publication}

Not applicable.

\section{Competing interests}

The authors declare that they have no competing interests.

\section{References}

1. Torre LA, Bray F, Siegel RL, Ferlay J, Lortet-Tieulent J and Jemal A: Global cancer statistics, 2012. CA Cancer J Clin 65: 87-108, 2015.

2. Li J, Feng Q, Wei X and Yu Y: MicroRNA-490 regulates lung cancer metastasis by targeting poly $\mathrm{r}(\mathrm{C})$-binding protein 1 . Tumour Biol 37: 15221-15228, 2016.

3. Ourari-Dhahri B, Ben Slima H, Ben Amar J, El Gharbi L, Ali M and Baccar Azzabi S: Management of non-small cell lung cancer. Tunis Med 90: 847-851, 2012.

4. Zamay TN, Zamay GS, Kolovskaya OS, Zukov RA, Petrova MM, Gargaun A, Berezovski MV and Kichkailo AS: Current and prospective protein biomarkers of lung cancer. Cancers 9, E155, 2017.

5. Lazzari C, Spreafico A,Bachi A, RoderH,Floriani I, Garavaglia D, Cattaneo A, Grigorieva J, Viganò MG, Sorlini C, et al: Changes in plasma mass-spectral profile in course of treatment of non-small cell lung cancer patients with epidermal growth factor receptor tyrosine kinase inhibitors. J Thorac Oncol 7: 40-48, 2012.

6. Tang Y, Qiao G, Xu E, Xuan Y, Liao M and Yin G: Biomarkers for early diagnosis, prognosis, prediction, and recurrence monitoring of non-small cell lung cancer. Onco Targets Ther 12: 4527-4534, 2017.

7. Lou W, Liu J, Gao Y, Zhong G, Chen D, Shen J, Bao C, Xu L, Pan J, Cheng J, et al: MicroRNAs in cancer metastasis and angiogenesis. Oncotarget 8: 115787-115802, 2017.

8. Zhou Q, Huang SX, Zhang F, Li SJ, Liu C, Xi YY, Wang L, Wang X, He QQ, Sun CC and Li DJ: MicroRNAs: A novel potential biomarker for diagnosis and therapy in patients with non-small cell lung cancer. Cell Prolif 50: 2017.

9. Krol J, Loedige I and Filipowicz W: The widespread regulation of microRNA biogenesis, function and decay. Nat Rev Genet 11: 597-610, 2010.

10. Esquela-Kerscher A and Slack FJ: Oncomirs-microRNAs with a role in cancer. Nat Rev Cancer 6: 259-269, 2006.

11. Lin PY, Yu SL and Yang PC: MicroRNA in lung cancer. Br J Cancer 103: 1144-1148, 2010.

12. Cui R, Meng W, Sun HL, Kim T, Ye Z, Fassan M, Jeon YJ, Li B, Vicentini C, Peng Y, et al: MicroRNA-224 promotes tumor progression in non-small cell lung cancer. Proc Natl Acad Sci USA 112: E4288-E4297, 2015.

13. Meng W, Ye Z, Cui R, Perry J, Dedousi-Huebner V, Huebner A, Wang Y, Li B, Volinia S, Nakanishi H, et al: MicroRNA-31 predicts the presence of lymph node metastases and survival in patients with lung adenocarcinoma. Clin Cancer Res 19: 5423-5433, 2013. 
14. Adams BD, Guo S, Bai H, Guo Y, Megyola CM, Cheng J, Heydari K, Xiao C, Reddy EP and Lu J: An in vivo functional screen uncovers miR-150-mediated regulation of hematopoietic injury response. Cell Rep 2: 1048-1060, 2012.

15. Wu Q, Jin H, Yang Z, Luo G, Lu Y, Li K, Ren G, Su T, Pan Y, Feng B, et al: MiR-150 promotes gastric cancer proliferation by negatively regulating the pro-apoptotic gene EGR2. Biochem. Biophys Res Commun 392: 340-345, 2010.

16. Zhang $\mathrm{N}$, Wei $\mathrm{X}$ and $\mathrm{Xu} \mathrm{L}$ : miR-150 promotes the proliferation of lung cancer cells by targeting P53. FEBS Lett 587: 2346-2351, 2013.

17. Cao M, Hou D, Liang H, Gong F, Wang Y, Yan X, Jiang X1, Wang C, Zhang J, Zen K, et al: miR-150 promotes the proliferation and migration of lung cancer cells by targeting SRC kinase signaling inhibitor 1. Eur J Cancer 50: 1013-1024, 2014.

18. Xu W, Jiang K, Shen M, Qian Y and Peng Y: SIRT2 suppresses non-small cell lung cancer growth by targeting JMJD2A. Biol Chem 396: 929-936, 2015.

19. Xu W, Jiang K, Shen M, Chen Y and Huang HY: Jumonji domain containing $2 \mathrm{~A}$ predicts prognosis and regulates cell growth in lung cancer depending on miR-150. Oncol Rep 35: 352-358. 2016

20. Orellana EA and Kasinski AL: MicroRNAs in cancer: A historical perspective on the path from discovery to therapy. Cancers 7 : $1388-1405,2015$
21. Frixa T, Donzelli S and Blandino G: Oncogenic MicroRNAs: Key players in malignant transformation. Cancers 7: 2466-2485, 2015.

22. Inamura $\mathrm{K}$ and Ishikawa Y: MicroRNA in lung cancer: Novel biomarkers and potential tools for treatment. J Clin Med 5: pii: E36, 2016.

23. Takahashi RU, Miyazaki $\mathrm{H}$ and Ochiya $\mathrm{T}$ : The roles of MicroRNAs in breast cancer. Cancers 7: 598-616. 2015.

24. He Y, Jiang $X$ and Chen J: The role of miR-150 in normal and malignant hematopoiesis. Oncogene 33: 3887-3893, 2014.

25. Huang S, Chen Y, Wu W, Ouyang N, Chen J, Li H, Liu X, Su F, Lin L and Yao Y: miR-150 promotes human breast cancer growth and malignant behavior by targeting the pro-apoptotic purinergic $\mathrm{P} 2 \mathrm{X}_{7}$ receptor. PLoS One 8: e80707, 2013.

26. Yokobori T, Suzuki S, Tanaka N, Inose T, Sohda M, Sano A Sakai M, Nakajima M, Miyazaki T, Kato H and Kuwano H: $M i R-150$ is associated with poor prognosis in esophageal squamous cell carcinoma via targeting the EMT inducer $Z E B 1$. Cancer Sci 104: 48-54, 2013.

27. Sun W, Zhang Z, Wang J, Shang R, Zhou L, Wang X, Duan J, Ruan B, Gao Y, Dai B, et al: MicroRNA-150 suppresses cell proliferation and metastasis in hepatocellular carcinoma by inhibiting the GAB1-ERK axis. Oncotarget 7: 11595-11608, 2016. 